\title{
The Bin Area Method: A Computationally Efficient Technique for Analysis of Ventricular and Atrial Intracardiac Electrograms
}

\author{
ROBERT D. THRONE, JANICE M. JENKINS, and LORENZO A. DICARLO* \\ From the Medical Computing Laboratory, Department of Electrical Engineering and Computer \\ Science, University of Michigan, Ann Arbor, and the *Cardiac Electrophysiology Laboratory, St. \\ Joseph Mercy Hospital of the Catherine McAuley Health Center, Ann Arbor, Michigan
}

THRONE, R.D., ET AL.: The Bin Area Method: A Computationally Efficient Technique for Analysis of Ventricular and Atrial Intracardiac Electrograms. Recent studies have reported a significant false positive rate in delivery of therapy by implantable antitachycardia devices utilizing detection algorithms based on sustained high rate. More selective decision schemes for the recognition of life-threatening arrhythmias have been recently proposed that use analysis of the intrinsic electrogram rather than rate alone. Morphological discrimination of abnormal electrograms using correlation waveform analysis (CWA) has been proposed as an effective method of intracardiac electrogram analysis, but its computational demands limit its use in implantable devices. A new method for intracardiac electrogram analysis, the bin area method (BAM), was created to detect abnormal cardiac conduction with computational requirements of one-half to one-tenth those of CWA. Like CWA, BAM is a template matching method that is sensitive to conduction changes revealed in the electrogram morphology and is independent of amplitude and baseline fluctuations. Performance of BAM and CWA were compared using bipolar right ventricular and right atrial electrode recordings from 47 patients undergoing clinical cardiac electrophysiology studies. Nineteen patients had 31 distinct monomorphic ventricular tachycardias (VTs) induced (group I), thirteen patients had paroxysmal bundle branch block of supraventricular origin (BBB) induced (group II), and 19 patients had retrograde atrial activation during right ventricular overdrive pacing (group III). (One patient was common to all three groups, and two patients were common to groups II and III.) Using the ventricular electrogram, both BAM and CWA distinguished VT from sinus rhythm in 28/31 (90\%) cases, and BBB from Normal Sinus Rhythm (NSR) in 13/13 (100\%) patients. Using the atrial electrogram, both $B A M$ and CWA distinguished anterograde from retrograde atrial activation in 19/19 (100\%) patients. BAM achieves similar performance to CWA with significantly reduced computational demands, and may make real-time analysis of intracardiac electrograms feasible for implantable pacemakers and antitachycardia devices. (PACE, Vol. 13, October 1990)

pacemaker-mediated tachycardia, dual-chamber pacemaker, tachycardia, tachyarrhythmia, retrograde atrial activation

\section{Introduction}

Recent studies have reported a significant false positive rate in delivery of therapy by im-

This work was partially supported by NSF grant No. EET-8351215 and a grant from Medtronic, Inc.

Address for reprints: Robert D. Throne, Medical Computing Laboratory, Department of Electrical Engineering, and Computer Science, 1301 Beal Street, Ann Arbor, MI 48109-2122.

Received March 29, 1990; Revision July 19, 1990; AcceptedJuly 19.1990 . plantable antitachycardia devices. ${ }^{1-7}$ Intracardiac electrogram analysis methods have been proposed for detecting one or more features of an atrial or ventricular electrogram for improved automated diagnosis of a specific arrhythmia, but few of these algorithms have been shown to be uniformly reliable. ${ }^{8-23}$

Correlation Waveform Analysis (CWA) has been proposed as an effective method of intracardiac waveform analysis for distinguishing ventricular electrograms during ventricular 
tachycardia (VT) from those during sinus rhythm, ${ }^{16,19,24,25}$ ventricular electrograms during normal sinus rhythm (NSR, QRS $\leq 120 \mathrm{msec}$ ) from those during paroxysmal bundle branch block of supraventricular origin (BBB), ${ }^{19}$ and retrograde atrial activation from anterograde atrial activation. ${ }^{26}$ CWA computes the correlation coefficient, $\rho$, between a normal template and each waveform under analysis. The correlation coefficient is independent of amplitude and baseline changes between the template and the signal under analysis, and produces a bounded index of merit between -1 and 1 (a value of 1 indicating a perfect match to the template). Although CWA is an effective method of intracardiac waveform analysis, it has the disadvantage of being a computationally demanding algorithm.

The purpose of the present study was to develop and test an innovative waveform analysis technique, the Bin Area Method (BAM), which has fewer computational demands than CWA but with a reliability similar to CWA. BAM compares corresponding areas, or bins, constructed from the template with bins constructed from subsequent depolarizations using a simple error measure. Like CWA, BAM was designed to be independent of amplitude and baseline fluctuations and to produce an index of merit between -1 and 1 where a value of 1 indicates a perfect match to the template.

In this study, BAM was used to compare ventricular electrograms during VT to those during sinus rhythm (or atrial fibrillation), ventricular electrograms during paroxysmal bundle branch block (BBB) of supraventricular origin to those during normal sinus rhythm (NSR), and atrial electrograms during 1:1 retrograde atrial activation. CWA was used as a standard against which to compare BAM for analysis of accuracy and computational requirements.

\section{Methods and Materials}

\section{Electrophysiology Study}

Bipolar $(1 \mathrm{~cm})$ atrial and ventricular endocardial electrograms were recorded during elective clinical cardiac electrophysiology studies as previously reported. ${ }^{16,19,26}$ Three $6 \mathrm{~F}$ quadrapolar electrode catheters with an interelectrode distance of $1 \mathrm{~cm}$ (USCI, Billerica, MA, USA) were introduced and advanced under fluoroscopic guidance to the high right atrium (or right atrial appendage) and right ventricular apex. Two catheters were positioned in the right ventricular apex with one dedicated to pacing and the other to obtaining recordings from the distal electrode pair. All recordings were made with the patients lying supine. Ventricular electrograms were recorded on FM magnetic tape (Hewlett-Packard Models 3968 and 3964A [Hewlett-Packard, San Diego, CA, USA]) from distal bipolar endocardial electrodes positioned in the right ventricular apex using amplifiers with filter settings of 0.5 to 500 $\mathrm{Hz}$ (Siemens Mingograf-7 [Siemens-Elema, Solna, Sweden]) or 1 to $500 \mathrm{~Hz}$ (Honeywell [Denver, CO, USA] Electronics for Medicine [Pleasantville, NY, USA]). Tape speed was $3 \frac{3}{4}$ inches/sec with a bandwidth of 0 to $1,250 \mathrm{~Hz}$. For comparing the results using CWA and BAM in discriminating ventricular electrograms during ventricular tachycardia from those during sinus rhythm or atrial fibrillation (group I), ventricular electrograms were obtained from 19 consecutive patients consisting of 17 men and two women. Specific details of this patient population are given in Table I. For comparing ventricular electrograms during normal sinus rhythm with those during paroxysmal bundle branch block of supraventricular origin (group II), ventricular electrograms were obtained from 13 consecutive patients consisting of six men and seven women. Specific details of this patient population are given in Table II. For examining atrial electrograms during retrograde and anterograde atrial activation (group III), atrial electrograms were obtained from 19 consecutive patients consisting of 12 men and seven women. None of the patients had dual atrioventricular nodal pathways or accessory atrioventricular connections, and specific details of these patients are given in Table III. As Tables I-III indicate, one patient was common to all three studies, and two patients were common to two studies. Recorded endocardial electrograms were subsequently replayed and digitized on a personal computer with a Tecmar Lab Master (Scientific Solutions, Inc., Solon, OH, USA) analog-to-digital system at a sampling rate of $1,000 \mathrm{~Hz}$. 
Table I.

Patient Data for Discriminating VT from SR/AF

\begin{tabular}{|c|c|c|c|c|c|}
\hline Patient & Sex & $\begin{array}{c}\text { Heart } \\
\text { Disease }\end{array}$ & Drugs & $\begin{array}{l}\text { Sinus Rhythm/ } \\
\text { Atrial Fibrillation } \\
\text { QRS Morphology }\end{array}$ & $\begin{array}{c}\text { Ventricular } \\
\text { Tachycardia } \\
\text { QRS Morphology }\end{array}$ \\
\hline 1 & $M$ & CAD & None & SR-Normal & RBB-S/R \\
\hline 2 & $M$ & CAD & None & SR-Normal & LBB-S/L \\
\hline $3 a$ & $M$ & CAD & None & SR-LBBB & LBB-S/R \\
\hline $3 b$ & & & & & LBB-S/L \\
\hline $4 a$ & $M$ & CAD & None & AF-LBBB & LBB-S/L \\
\hline $4 b$ & & & & & LBB-S/L \\
\hline $5 a$ & $M$ & CAD & Proc & SR-Normal & RBB-I/R \\
\hline $5 b$ & & & & & LBB-I/R \\
\hline $6 a$ & $M$ & CAD & Proc & SR-Normal & RBB-I/L \\
\hline $7 a$ & $\mathrm{~F}$ & CAD & $\mathrm{Am}$ & SR-Normal & RBB-S/L \\
\hline $7 b$ & & & & & RBB-S/L \\
\hline 8 & $\mathrm{~F}$ & None & $\mathrm{Am}$ & SR-Normal & RBB-S/R \\
\hline 9 & $M$ & CAD & Qu Me & SR-Normal & LBB-S/L \\
\hline $10 a$ & $M$ & CAD & $\mathrm{Am}$ & SR-LBBB & LBB-S/L \\
\hline 11 & $M$ & VHD & $\mathrm{Am}$ & SR-LBBB & LBB-S/R \\
\hline 12 & $M$ & CAD & $\mathrm{Am} \mathrm{Me}$ & SR-LBBB & LBB-I/R \\
\hline 13 & $M$ & CAD & En & SR-LBBB & LBB-I/L \\
\hline 14 & $M$ & CAD & Qu Me & SR-RBBB & RBB-S/R \\
\hline 15 & $M$ & CAD & Proc & SR-RBBB & LBB-S/R \\
\hline $16 a$ & $M$ & CAD & $\mathrm{Am}$ & SR-IVCD & RBB-I/R \\
\hline $16 b$ & & & & & RBB-S/L \\
\hline 17 & $M$ & CAD & $\mathrm{Am}$ & SR-IVCD & LBB-S/L \\
\hline $18 a$ & $M$ & CAD & $\mathrm{Am}$ & SR-IVCD & †RBB-S/R \\
\hline $18 b$ & & & & & †LBB-S/L \\
\hline $18 \mathrm{c}$ & & & & & †LBB-S/L \\
\hline $18 d$ & & & & & †RBB-S/R \\
\hline $18 e$ & & & & & †RBB-S/R \\
\hline $10 b^{\star}$ & $M$ & CAD & Am En & SR-IVCD & RBB-S/R \\
\hline $10 c$ & & & & & LBB-I/L \\
\hline $6 b^{\star}$ & $M$ & CAD & En Proc & AF-IVCD & RBB-S/R \\
\hline 19 & $M$ & CAD & Qu Di & AF-IVCD & RBB-S/L \\
\hline
\end{tabular}

These patients were studied on two different days with two different VT morphologies. TThis patient had five distinct VT morphologies. CAD = coronary artery disease; VHD = valvular heart disease; $\mathrm{Am}=$ amiodarone; $\mathrm{Di}$ = digoxin; $\mathrm{En}=$ encainide; $\mathrm{Me}=$ mexiletene, $\mathrm{Proc}=$ procainamide; $\mathrm{Qu}=$ quinidine; $\mathrm{SR}=$ sinus rhythm; $\mathrm{AF}=$ atrial fibrillation; LBBB = left bundle branch block; RBBB = right bundle branch block; IVCD = nonspecific intraventricular conduction delay; $\mathrm{LBB}=$ left bundle branch morphology; RBB = right bundle branch morphology; I = inferior axis; $\mathrm{S}=$ superior axis; $\mathrm{L}=$ leftward; $\mathrm{R}=$ rightward.

\section{Method of Analysis}

Data sets consisted of three passages from each patient. For discriminating ventricular electrograms during VT from those during sinus rhythm or atrial fibrillation (SR/AF), two separate ventricular passages were digitized from re- cordings made during SR/AF, and a third passage was digitized during induced monomorphic VT. For discriminating paroxysmal bundle branch block of supraventricular origin from normal sinus rhythm, two separate ventricular passages were digitized from recordings made during NSR. The third passage was digitized from recordings 
Table II.

Patient Data for Discriminating Paroxysmal Bundle Branch Block of Supraventricular Origin from Normal Sinus Rhythm

\begin{tabular}{|c|c|c|c|c|c|}
\hline Patient & Sex & $\begin{array}{c}\text { Heart } \\
\text { Disease }\end{array}$ & Drugs & $\begin{array}{c}\text { Method of } \\
\text { BBB Induction }\end{array}$ & $\begin{array}{c}\text { Aberration } \\
\text { Morphology }\end{array}$ \\
\hline 20 & $\mathrm{~F}$ & None & None & Spontaneous & RBBB \\
\hline 21 & $\mathrm{~F}$ & None & None & AOP & RBBB \\
\hline 22 & $\mathrm{~F}$ & None & None & AOP & RBBB \\
\hline 8 & $\mathrm{~F}$ & None & None & Spontaneous & RBBB \\
\hline 23 & $\mathrm{~F}$ & None & Iso & AOP & RBBB \\
\hline 24 & $\mathrm{~F}$ & None & Iso & Atrial Fibrillation & RBBB \\
\hline 25 & $\mathrm{~F}$ & None & Iso & Spontaneous & RBBB \\
\hline 26 & $M$ & COPD & None & AOP & RBBB \\
\hline 27 & $M$ & CAD & None & Spontaneous & RBBB \\
\hline 28 & $M$ & CAD & None & AOP & RBBB \\
\hline 29 & $M$ & CAD & Proc & AOP & RBBB \\
\hline 30 & $M$ & None & Iso & AOP & LBBB \\
\hline 31 & $M$ & CAD & None & AOP & LBBB \\
\hline
\end{tabular}

$\mathrm{CAD}=$ coronary artery disease; $\mathrm{COPD}=$ chronic obstructive pulmonary disease; Iso $=$ isopril; Proc $=$ procainamide; LBBB = left bundle branch block; RBBB = right bundle branch block; AOP = atrial overdrive pacing.

Table III.

Patient Data for Discriminating Retrograde from Anterograde Atrial Activation

\begin{tabular}{|c|c|c|c|c|}
\hline Patient & Sex & $\begin{array}{c}\text { Heart } \\
\text { Disease }\end{array}$ & Drugs & $\begin{array}{c}\text { Atrial Electrode } \\
\text { Location }\end{array}$ \\
\hline 23 & $\mathrm{~F}$ & None & Iso & RAA \\
\hline 32 & $M$ & None & None & RAA \\
\hline 33 & $M$ & None & None & RAA \\
\hline 34 & $\mathrm{~F}$ & None & None & RAA \\
\hline 35 & $M$ & CAD & None & RAA \\
\hline 36 & $M$ & None & None & RAA \\
\hline 37 & $M$ & None & None & RAA \\
\hline 38 & $\mathrm{~F}$ & None & None & RAA \\
\hline 26 & $M$ & COPD & None & RAA \\
\hline 39 & $\mathrm{~F}$ & None & None & RAA \\
\hline 40 & $M$ & CAD & None & RAA \\
\hline 41 & $M$ & CAD & $\mathrm{Ve}$ & RAA \\
\hline 8 & $\mathrm{~F}$ & None & None & RAA \\
\hline 42 & $M$ & None & None & RAA \\
\hline 43 & $\mathrm{~F}$ & None & None & HRA \\
\hline 44 & $M$ & None & Qu Ve & HRA \\
\hline 45 & $M$ & None & None & HRA \\
\hline 46 & $\mathrm{~F}$ & None & Ep & HRA \\
\hline 47 & $M$ & None & En & HRA \\
\hline
\end{tabular}

$\mathrm{En}=$ encainide; $\mathrm{Ep}=$ epinephrine; Iso = isopril; $\mathrm{Qu}=$ quinidine; $\mathrm{Ve}=$ verapamil; $\mathrm{CAD}=$ coronary artery disease; $\mathrm{COPD}=$ chronic obstructive pulmonary disease; RAA = right atrial appendage; HRA = high right atrium. 
made during BBB. For discriminating retrograde from anterograde atrial activation, two separate atrial passages were digitized from recordings made during sinus rhythm (anterograde atrial activation) and a third passage during retrograde atrial activation while pacing the right ventricle at a rate sufficient to maintain 1:1 retrograde conduction to the atrium.

In all cases, the initial passage of SR/AF, NSR, or anterograde atrial activation was used to construct a ventricular or atrial electrogram template by signal averaging. The template was employed for subsequent comparison with a second separate SR/AF, NSR, or anterograde control passage and a later passage of either VT (group I), BBB (group II), or retrograde atrial activation (group III). A careful selection of window size effectively excluded any local repolarization in order to avoid the inclusion of injury current caused by temporary endocardial damage adjacent to the catheter. ${ }^{19,26} \mathrm{~A}$ software trigger (peak detector) was used for automatic recognition of each of the sequential waveforms. ${ }^{16,19,26}$ An algorithm was declared successful if there was no overlap in the ranges of the similarity values $p$ during the abnormal passage when compared to the ranges during the normal passage.

In presenting both CWA and BAM, the following notation will be used.

$\mathrm{N}=$ the number of points in the template.

$t_{i}=$ the template points.

$\mathrm{S}_{\mathrm{i}}=$ the signal points to be processed.

$\overline{\mathrm{t}}=$ the template average.

$\overline{\mathrm{s}}=$ the signal average.

\section{CWA}

The correlation coefficient, $\rho,^{16,19,26}$ is independent of amplitude and baseline fluctuations between the template and waveform under analysis and produces an output between -1 and 1 . Mathematically, the correlation coefficient is defined as:

$$
\rho=\frac{\sum_{i=1}^{i=N}\left(t_{i}-\bar{t}\right)\left(s_{i}-\bar{s}\right)}{\sqrt{\sum_{k=1}^{k=N}\left(t_{k}-\bar{t}\right)^{2}} \sqrt{\sum_{k=1}^{k=N}\left(s_{k}-\bar{s}\right)^{2}}}
$$

For comparison with the similarity measure used in BAM, the correlation coefficient is equivalent to the following squared-error norm:

$$
\rho=1-\frac{1}{2} \sum_{i=1}^{i=N}\left(\frac{t_{i}-\bar{t}}{\sqrt{\sum_{k=1}^{k=N}\left(t_{k}-\bar{t}\right)^{2}}}-\frac{s_{i}-\bar{s}}{\sqrt{\sum_{k=1}^{k=N}\left(s_{k}-\bar{s}\right)^{2}}}\right)^{2}
$$

To avoid the square-root computation, we can equivalently compute

$$
\mu=\rho^{2} \operatorname{sign}(\rho)
$$

where sign returns the arithmetic sign of the argument $( \pm 1)$.

\section{BAM}

BAM compares corresponding areas or bins constructed from the template with bins constructed from subsequent depolarizations using a simple error measure. Consecutive sample points are summed to estimate the areas using a rectangular area rule in equal sized bins. The average of these bin values (DC offset) is then removed resulting in a correction of baseline shift, and then these corrected bin values are normalized by the absolute sum of all corrected bin values. As a final step, the sum of the absolute difference of these normalized and corrected bins with an identically processed template is computed.

To compute bin areas, we add consecutive sample points. To form 5-point bins,

$$
\begin{aligned}
\mathrm{S}_{1} & =\mathrm{s}_{1}+\mathrm{s}_{2}+\mathrm{s}_{3}+\mathrm{s}_{4}+\mathrm{s}_{5} \\
\mathrm{~S}_{2} & =\mathrm{s}_{6}+\mathrm{s}_{7}+\mathrm{s}_{8}+\mathrm{s}_{9}+\mathrm{s}_{10} \\
& \vdots \\
\mathrm{S}_{\mathrm{M}} & =\mathrm{s}_{\mathrm{N}-4}+\mathrm{s}_{\mathrm{N}-3}+\mathrm{s}_{\mathrm{N}-2}+\mathrm{s}_{\mathrm{N}-1}+\mathrm{s}_{\mathrm{N}}
\end{aligned}
$$

To form three-point bins,

$$
\begin{aligned}
\mathrm{S}_{1} & =\mathrm{s}_{1}+\mathrm{s}_{2}+\mathrm{s}_{3} \\
\mathrm{~S}_{2} & =\mathrm{s}_{4}+\mathrm{s}_{5}+\mathrm{s}_{6} \\
& \vdots \\
\mathrm{S}_{\mathrm{M}} & =\mathrm{s}_{\mathrm{N}-2}+\mathrm{s}_{\mathrm{N}-1}+\mathrm{s}_{\mathrm{N}}
\end{aligned}
$$

To form one-point bins, $S_{i}=s_{i}, i=1, \ldots, N$. Template points, $t_{i}$, are processed similarly to form the $T_{i}$. For M equally sized bins in the template, the index of merit for BAM is given as

$$
\begin{aligned}
& \quad \mu=1-\sum_{\mathrm{i}=1}^{\mathrm{i}=\mathrm{M}}\left|\frac{\mathrm{T}_{\mathrm{i}}-\overline{\mathrm{T}}}{\sum_{\mathrm{k}=1}^{\mathrm{k}=\mathrm{M}}\left|\mathrm{T}_{\mathrm{k}}-\overline{\mathrm{T}}\right|}-\frac{\mathrm{S}_{\mathrm{i}}-\overline{\mathrm{S}}}{\sum_{\mathrm{k}=1}^{\mathrm{k}=\mathrm{M}}\left|\mathrm{S}_{\mathrm{k}}-\overline{\mathrm{S}}\right|}\right| \\
& \text { where } \overline{\mathrm{S}}=\frac{1}{\mathrm{M}} \sum_{\substack{\mathrm{k}=1 \\
\mathrm{k}=\mathrm{M}}} \mathrm{S}_{\mathrm{k}} \text {, and } \overline{\mathrm{T}}=\frac{1}{\mathrm{M}} \sum_{\substack{k=1 \\
\mathrm{k}=\mathrm{M}}} \mathrm{T}_{\mathrm{k}} .
\end{aligned}
$$


Table IV.

\begin{tabular}{|c|c|c|c|c|c|}
\hline \multirow[b]{2}{*}{ Alignment } & \multirow[b]{2}{*}{ CWA } & \multicolumn{3}{|c|}{ BAM } & \multirow{2}{*}{$\begin{array}{c}\text { Total } \\
\text { Tested }\end{array}$} \\
\hline & & $1 \mathrm{pt}$ & 3 pt & $5 \mathrm{pt}$ & \\
\hline \multicolumn{6}{|c|}{ VT vs. SR/AF (Group I) } \\
\hline Original (Peak) & 27 & 26 & 25 & 25 & 31 \\
\hline Best Fit & 28 & 28 & 28 & 26 & 31 \\
\hline \multicolumn{6}{|c|}{ BBB vs. NSR (Group II) } \\
\hline Original (Peak) & 13 & 13 & 13 & 13 & 13 \\
\hline Best Fit & 13 & 13 & 13 & 13 & 13 \\
\hline \multicolumn{6}{|c|}{ RAA vs. AAA (Group III) } \\
\hline Original (Peak) & 14 & 15 & 16 & 14 & 19 \\
\hline Best Fit & 19 & 19 & 19 & 19 & 19 \\
\hline
\end{tabular}

Because of the design of BAM, all template processing is performed in advance, i.e., prior to analysis of incoming depolarizations subject to analysis. BAM requires one-half the multiplications of CWA for 1-point bins, one-sixth the multiplications of CWA for three-point bins, and onetenth the multiplications for five-point bins. Note that we need to compute $1 / \sum_{k=1}^{k=M}\left|S_{k}-\bar{S}\right|$ only once. After that we only need to do multiplications which involve less computation time than divisions in the type of microprocessors likely to be used in an implantable device. BAM is similar to area of difference (AD), ${ }^{13,14,18}$ but is designed to be independent of amplitude and baseline fluctuations.

\section{Triggering}

The true (ideal) performance for any template matching algorithm requires precise alignment between the template and current waveform under analysis. However, in an implantable device a peak detector or slope detector with alignment at a single point is more likely. In order to examine the effect of trigger alignment on performance, BAM and CWA were computed using both a peak amplitude trigger (original) and a best fit alignment between template and waveform under analysis within an $11 \mathrm{msec}$ window of the original trigger location as previously described. ${ }^{19,26}$

\section{Results}

For either method, BAM or CWA, there is no universal threshold which will separate VT from SR/AF, BBB from NSR, or retrograde from anterograde atrial activation in the patient population studied. Instead, patient specific thresholds were required for each method. Results for groups I, II, and III are summarized in Table IV for both the original and the best fit triggers. In some instances, while there was no overlap in the ranges and an algorithm was declared successful, the ranges of similarity measures during the abnormal and corresponding normal passages were very close.

\section{Detecting Ventricular Tachycardia (Group I)}

Figure 1 summarizes results using BAM with three-point bins (panel A) and CWA (panel B) at 
THRONE, ET AL.

A

PATIENT

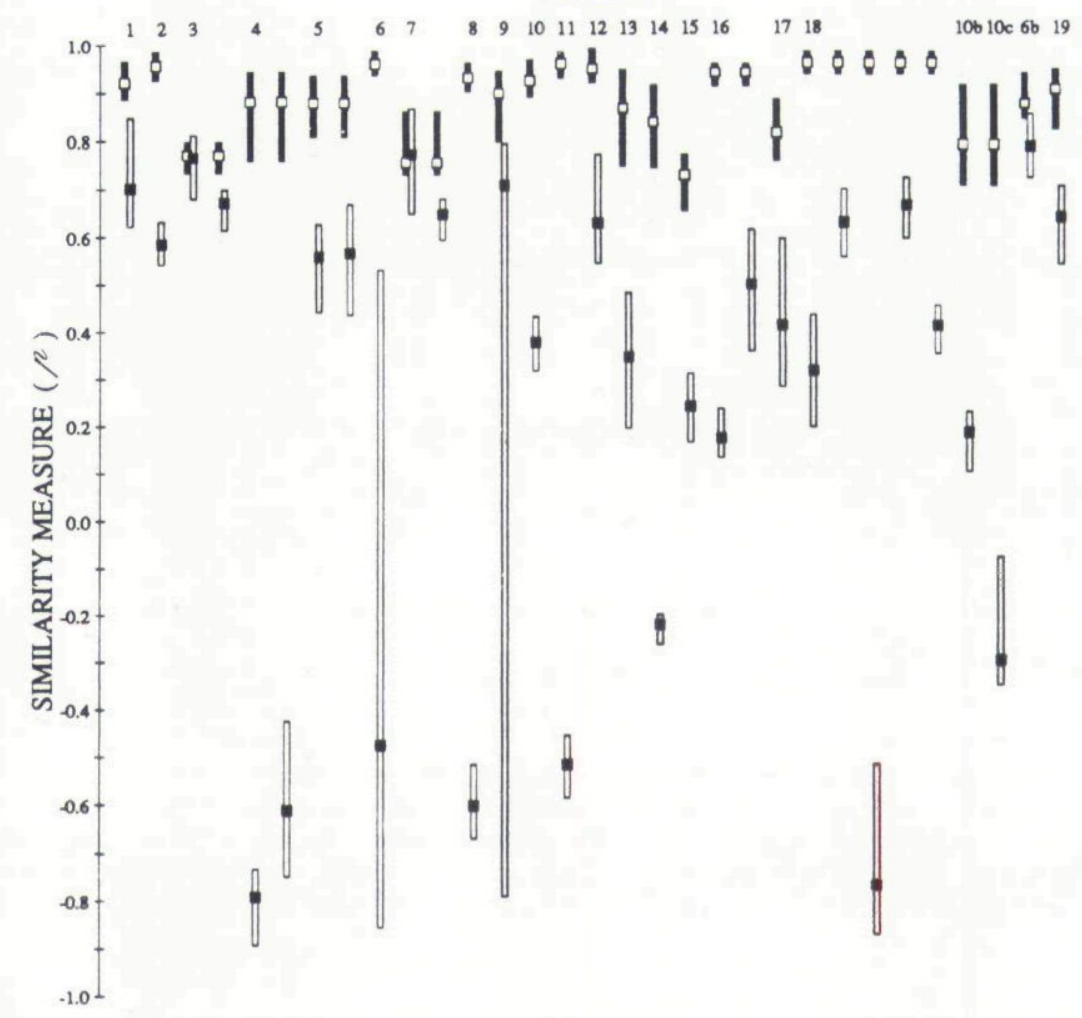

B

PATIENT

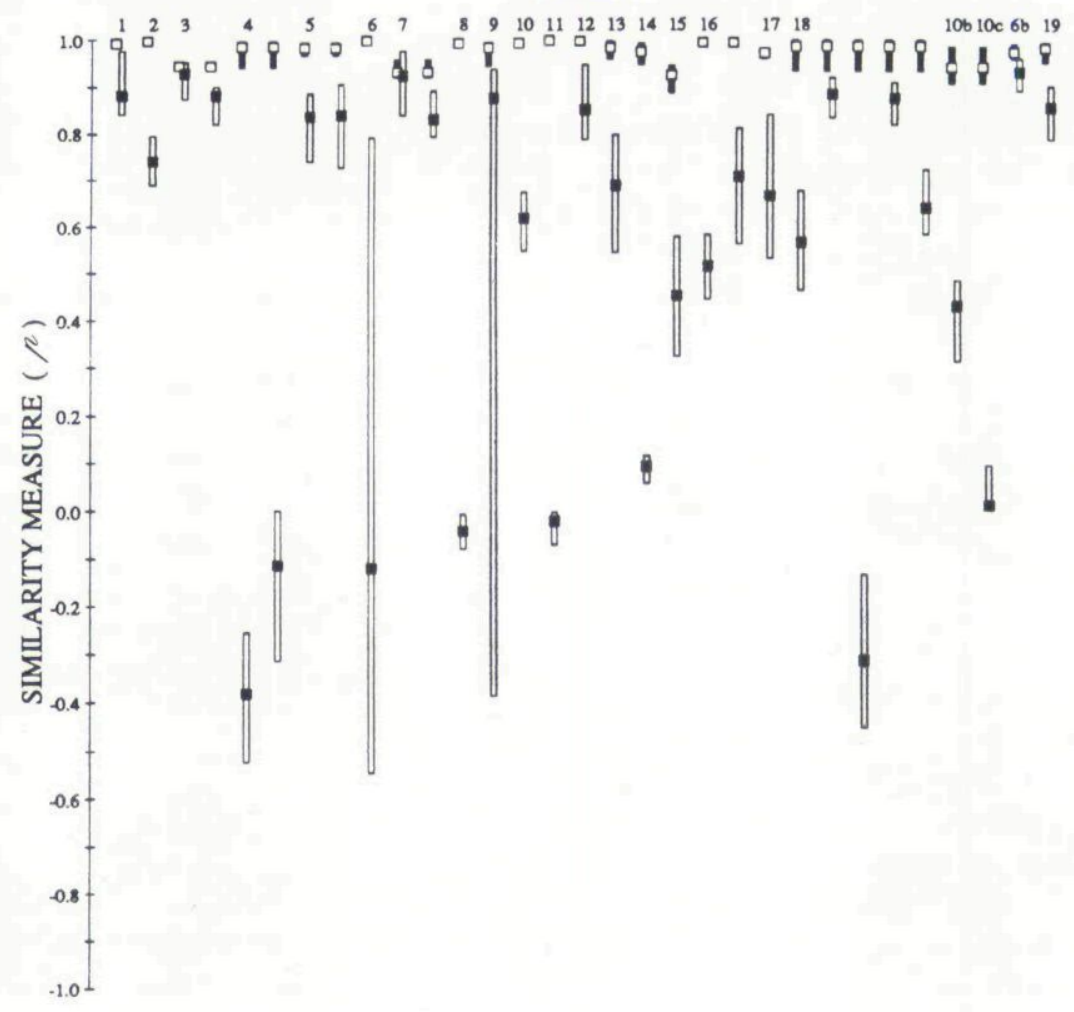



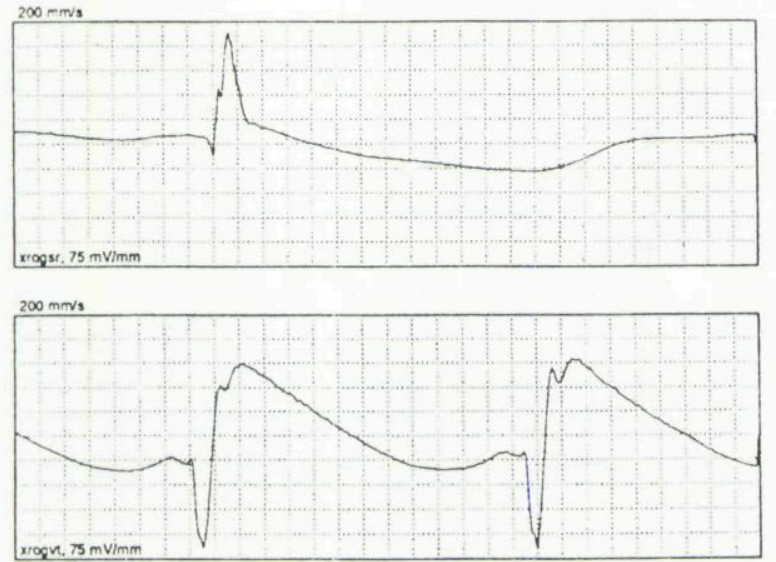

Figure 2. Ventricular electrograms during sinus rhythm (top) and ventricular tachycardia (bottom) for patient 8. Both electrograms are displayed at $200 \mathrm{~mm} /$ sec and at the same scale. (The dotted lines represent $5 \mathrm{~mm}$.) Both BAM and CWA could easily distinguish all ventricular tachycardia depolarizations from all sinus rhythm depolarizations for this patient.
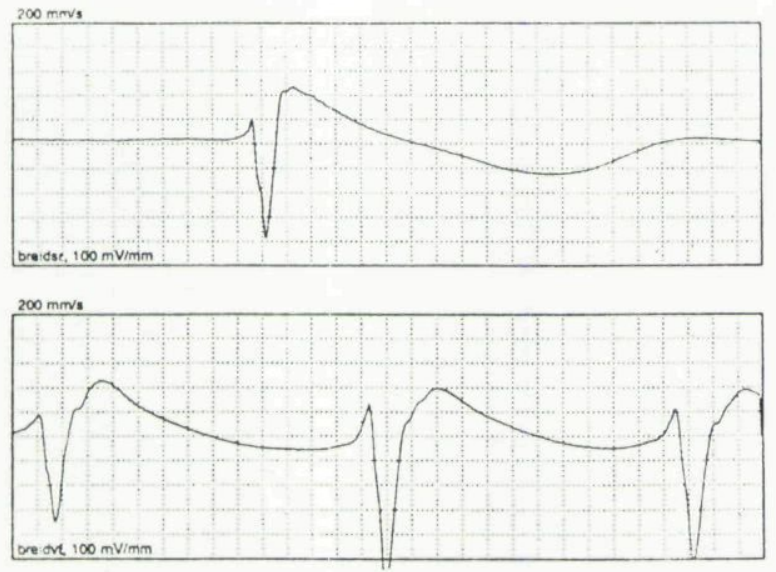

Figure 3. Ventricular electrograms during sinus rhythm (top) and ventricular tachycardia (bottom) for patient 12. Both electrograms are displayed at $200 \mathrm{~mm} /$ sec and at the same scale. (The dotted lines represent 5 $\mathrm{mm}$.) Both BAM and CWA could distinguish all ventricular tachycardia depolarizations from all sinus rhythm depolarizations for this patient.
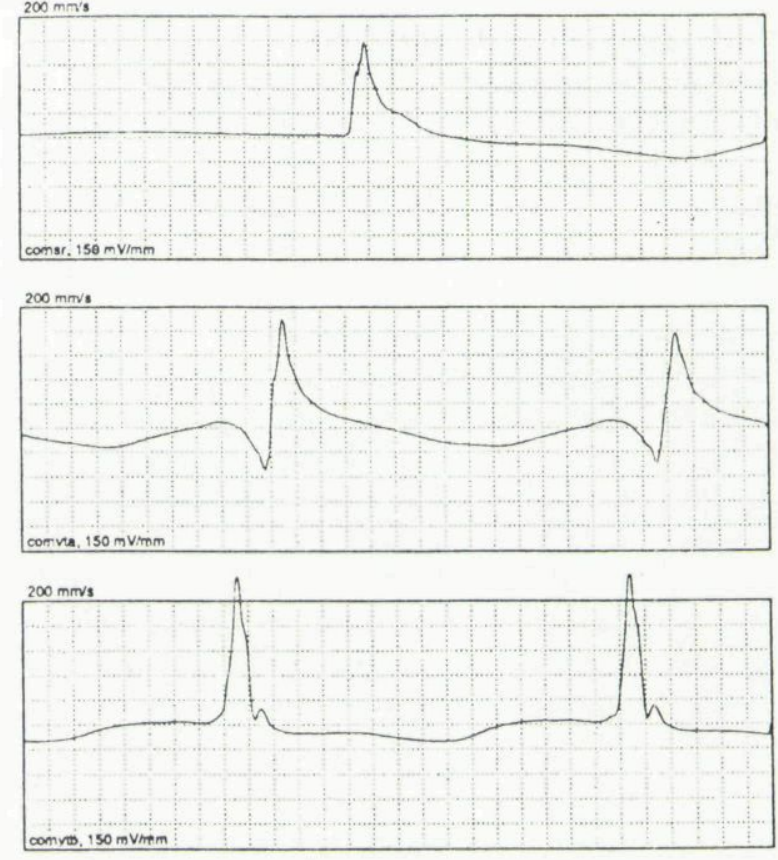

Figure 4. Ventricular electrograms during sinus rhythm (top) and two distinct ventricular tachycardia morphologies for patient 7. Both electrograms are displayed at $200 \mathrm{~mm} / \mathrm{sec}$ and at the same scale. (The dotted lines represent $5 \mathrm{~mm}$.) Both BAM and CWA failed to distinguish all of the ventricular tachycardia depolarizations during the passage of the first VT morphology (middle passage), but both distinguished all depolarizations of the second VT from sinus rhythm.

the best fit location in discriminating VT from $\mathrm{SR} / \mathrm{AF}$. Using the original trigger, BAM with one-, three-, and five-point bins discriminated VT from $\mathrm{SR} / \mathrm{AF}$ in $26 / 31(84 \%), 25 / 31(81 \%), 25 / 31(81 \%)$ cases, while CWA discriminated VT from SR/AF in $27 / 31(87 \%)$ cases. Using the best fit alignment, BAM with one-, three- and five-point bins discriminated VT from SR/AF in $28 / 31(90 \%)$, and $28 / 31(90 \%)$, and $26 / 31(84 \%)$ instances, while CWA discriminated VT from SR/AF in 28/31 $(90 \%)$ cases.

Figures 2, 3, and 4 display ventricular electrograms during both sinus rhythm and mono-

Figure 1. Results of BAM with three-point bins (A) and CWA (B) using the best fit alignment for distinguishing VT from SR (group I). The ranges of $p$ during SR/AF are shown in white, with a black box at the mean, while the ranges of $n$ for VT are shown in black, with a white box at the mean. 
morphic ventricular tachycardia for patients 8 , 12, and 7. All electrograms are played at $200 \mathrm{~mm} / \mathrm{sec}$ and at the same scale. For patient 8 (Fig. 2) the electrograms during both sinus rhythm and VT are easily distinguishable for both BAM and CWA. For patient 12 (Fig. 3), both BAM and CWA could distinguish VT from SR, even though the electrograms appear quite similar. For patient 7 (Fig. 4), both BAM and CWA could not distinguish the first VT from SR, but both could distinguish the second VT. While it may appear that the ventricular electrograms during the first VT look different than during SR, the template was chosen based on the SR depolarization only, and was chosen to begin when SR depolarization began. Thus the earlier activation during the first VT was not included.

A

PATIENT

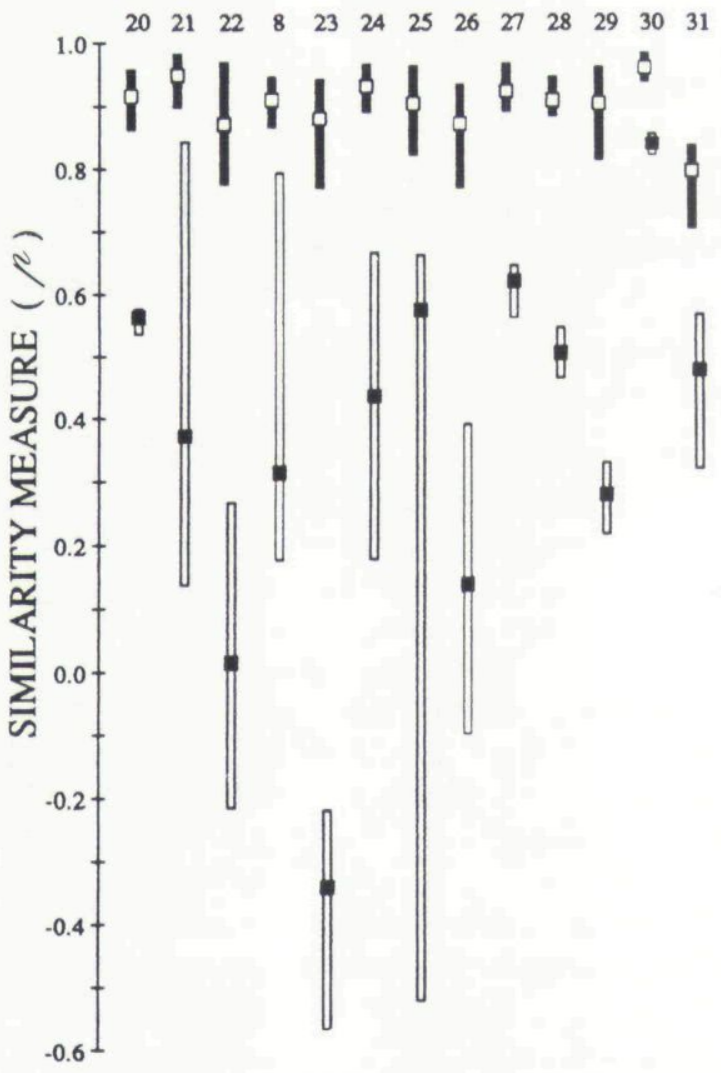

\section{Detecting Paroxysmal Bundle Branch Block (Group II)}

Figure 5 summarizes results of using BAM with three-point bins (panel A) and CWA (panel $\mathrm{B}$ ) at the best fit location in discriminating BBB from NSR. Using the original trigger, both BAM with one-, three-, and five-point bins and CWA discriminated BBB from NSR in 13/13 (100\%) patients. Using the best fit alignment, both BAM with one-, three-, and five-point bins and CWA discriminated BBB from NSR in 13/13 (100\%) patients.

\section{Detecting Retrograde Atrial Activation (Group III)}

Figure 6 summarizes results using both BAM with three-point bins (Panel A) and CWA (panel

B

\section{PATIENT}

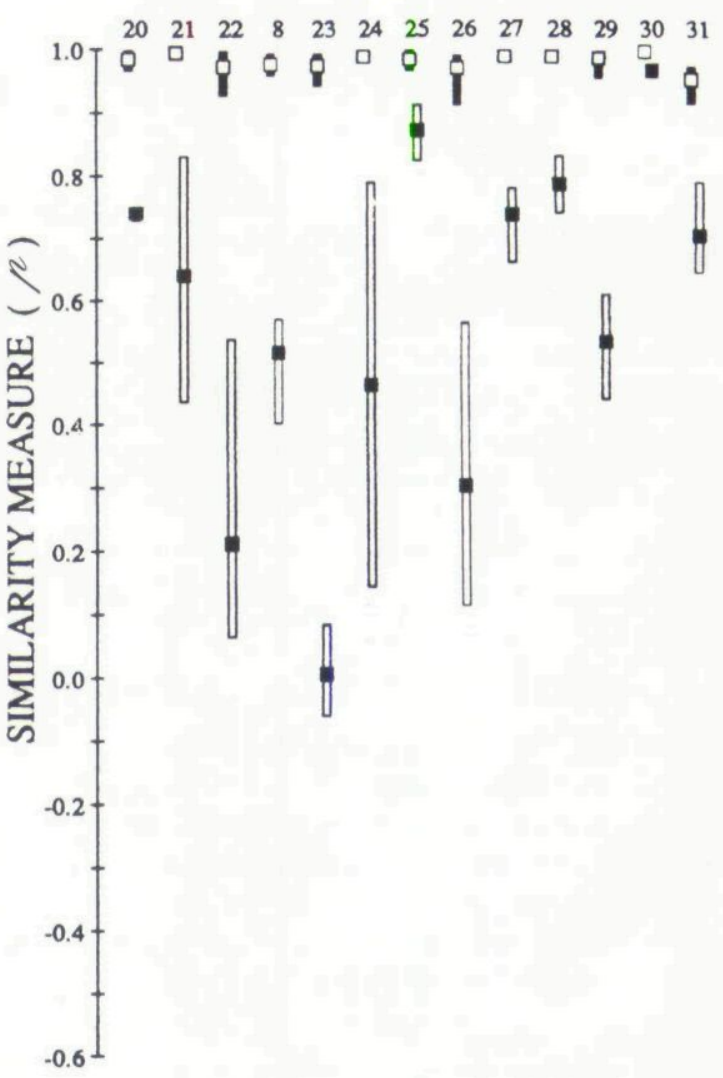

Figure 5. Results of BAM with three-point bins (A) and CWA (B) using the best fit alignment for distinguishing BBB from NSR (Group II). The ranges of $p$ during NSR are shown in white, with a black box at the mean, while the ranges of $p$ for BBB are shown in black, with a white box at the mean. 
A

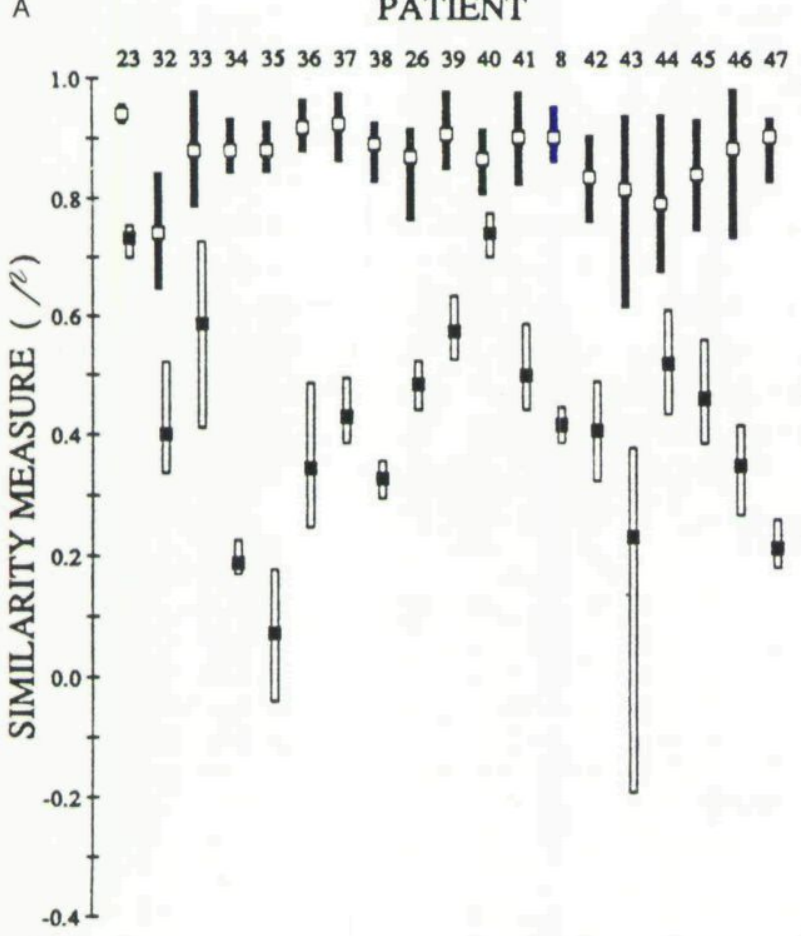

B

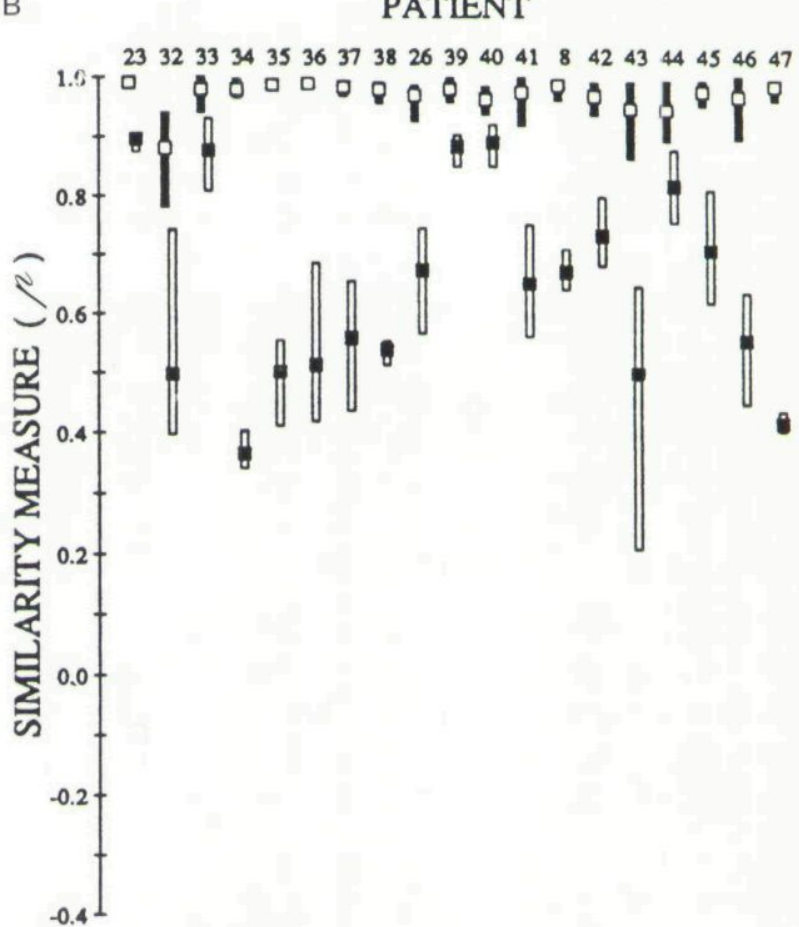

Figure 6. Results of BAM with 3-point bins (A) and CWA (B) using the best fit alignment for distinguishing RAA from AAA (group III). The ranges of $\rho$ during anterograde atrial activation (AAA) are shown in white, with a black box at the mean, while the ranges of $n$ for retrograde atrial activation (RAA) are shown in black, with a white box at the mean.

B) using the best fit location for separating retrograde atrial activation from anterograde atrial activation. Using the original trigger BAM with one-, three-, and five-point bins discriminated anterograde from retrograde atrial activation in $15 / 19$ (79\%), 16/19 (84\%), and 14/19 (74\%) patients, while CWA discriminated anterograde from retrograde ranges in $14 / 19(74 \%)$ patients. Using the best fit alignment, both BAM with one-, three-, and five-point bins and CWA discriminated retrograde from anterograde atrial activation in $19 / 19(100 \%)$ patients.

\section{Discussion}

Overall, the results for BAM in discriminating VT from sinus rhythm/atrial fibrillation, BBB from normal sinus rhythm, and retrograde from anterograde atrial activation, were similar to those of CWA. In detecting VT, CWA was slightly superior to BAM at the original trigger location. The results using BAM with one- and three-point bins were identical to those of CWA at the best fit location. In discriminating BBB from NSR, both BAM with one-, three-, and five-point bins and CWA were successful in $13 / 13(100 \%)$ cases at both the original and best fit trigger locations. In detecting retrograde from anterograde atrial activation, BAM with one-, and three-point bins was slightly superior to CWA at the original trigger. Both methods detected all retrograde atrial activation using the best fit trigger location.

As indicated in Figures 1, 5, and 6, ranges of values for CWA are generally smaller than those using BAM, while mean values are generally better separated using BAM than CWA. Given the reduced computational requirements of BAM, this separation of the mean values (typically used as a performance measure) is an important consideration.

While CWA has been successful in discriminating VT from sinus rhythm, ${ }^{16,19,24}$ paroxysmal BBB of supraventricular origin from normal sinus rhythm, ${ }^{19}$ and retrograde from anterograde atrial 
activation, ${ }^{26}$ it has the potential disadvantage of being too computationally demanding for use in an implantable device. One alternative method, $\mathrm{AD},{ }^{13,14,18}$ has been shown to discriminate ventricular electrograms during VT from those during sinus rhythm, and to differentiate ventricular electrograms during paroxysmal bundle branch block from those of sinus rhythm with a normal QRS $^{19}$ with much lower computational cost than CWA. However, in contrast to CWA and BAM, the results using $\mathrm{AD}$ may be adversely affected by amplitude changes. ${ }^{18}$

\section{Conclusion}

Rapid and reliable digital analysis of intracardiac electrograms may significantly increase the specificity of detection of pathological tachycardia by implantable antitachycardia devices without sacrificing sensitivity. We present the

\section{References}

1. Echt DS, Armstrong K, Schmidt P, et al. Clinical experience, complications, and survival in 70 patients with the automatic implantable cardioverter/defibrillator. (abstract) Circulation 1985; 71:289.

2. Manz M, Gerckens U, Luderitz B. Erroneous discharge from an implanted automatic cardioverter/defibrillator during supraventricular tachyarrhythmia-induced ventricular fibrillation. Am J Cardiol 1986; 7:343.

3. Platia EV, Griffith LSC, Reid PR, et al. Complications with the automatic implantable cardioverter defibrillator. (abstract) J Am Coll Cardiol 1986; 2:200.

4. Gabry MD, Brodman R, Johnston D, et al. Automatic implantable cardioverter-defibrillator: Patient survival, battery longevity, and shock delivery analysis. J Am Coll Cardiol 1987; 9:1349-1356.

5. Podczeck A, Frohner K, Heif CH, et al. Termination of life-threatening ventricular tachyarrhythmias by the automatic implantable cardioverter defibrillator (AICD). (abstract) PACE 1989; 12:1256.

6. Veltri E, Mower M, Aarons D, et al. Automatic implantable cardioverter-defibrillator: Comparison of patients with and without discharge. (abstract) Circulation 1989; 80:530.

7. Steinberg JS, Sugalski JS, Haratonic K. Cardiac rhythm precipitating automatic-implantable cardioverter defibrillator discharge in outpatients: Observations from transtelephonic recordings. (abstract) Circulation 1989; 80:530.

8. McAlister H, Klementowicz PT, Calderon EM, et design of a new algorithm, BAM which like CWA is a template matching technique independent of amplitude or baseline fluctuations between the template and each waveform under analysis. BAM has been shown to be reliable in intracardiac waveform analysis, and compares favorably with correlation waveform analysis at considerably reduced complexity. Depending on the size of the bins used, BAM requires from one-half to one-tenth the multiplications of CWA. BAM may make real-time morphology analysis of intracardiac electrograms feasible for antitachycardia devices.

Acknowledgments: The authors wish to express their appreciation to Colleen Hoover, B.S., Barbara Nagrant, C.V.R.T., and Judy Jaffke, R.T. (R), for their technical assistance in the cardiac electrophysiology laboratory, and Ms. Debbie Laird for preparation of this manuscript.

al. Atrial electrogram analysis: Antegrade vs retrograde. PACE 1988; 11:1703-1707.

9. Pannizzo F, Furman S. Automatic discrimination of retrograde $\mathrm{P}$ waves for dual chamber pacemakers. (abstract) J Am Coll Cardiol 1985; 5:393.

10. Pannizzo F, Amikam S, Bagwell P, et al. Discrimination of antegrade and retrograde atrial depolarization by electrogram analysis. Am Heart J 1986; 112:780-786.

11. Bernheim C, Markewitz A, Kemes BH. Can reprogramming of atrial sensitivity avoid an endless loop tachycardia? (abstract) PACE 1986; 4:18.

12. McAlister H, Adelson R, Calderon E, et al. Atrial electrogram analysis: Antegrade vs retrograde. (abstract) PACE 1988; 11:818.

13. Tomaselli G, Nielsen A, Finke W, et al. Morphologic differences of the endocardial electrogram in beats of sinus and ventricular origin. PACE 1988; $11: 254-262$.

14. Langberg J, Gibb W, Auslander D, et al. Identification of ventricular tachycardia with use of the morphology of the endocardial electrogram. Circulation 1988; 77:1363-1369.

15. Timmis G, Westveer D, Bakalyar D, et al. Discrimination of antegrade from retrograde atrial electrograms for physiologic pacing. PACE 1988; 11:130140.

16. Lin D, DiCarlo LA, Jenkins JM. Identification of ventricular tachycardia using intracavitary ventricular electrograms: Analysis of time and frequency domain patterns. PACE 1988; 11:15921606.

17. Pannizzo F, Furman S. Frequency spectra of ven- 
tricular tachycardia and sinus rhythm in human intracardiac electrograms-application to tachycardia detection for cardiac pacemakers. IEEE Trans Biomed Eng 1988; 35:421-425.

18. Tomaselli G, Gibb W, Langberg J, et al. In vivo testing of a morphology based approach to cardiac rhythm identification. (abstract) Circulation 1987; 76:1116.

19. Throne RD, DiCarlo LA, Jenkins JM, et al. Paroxysmal bundle branch block of supraventricular origin: A possible source of misdiagnosis in detecting ventricular tachycardia using time domain analyses of intraventricular electrograms. PACE 1990; 13:453-468.

20. Wainwright RJ, Davies DW, Tooley MA. Ideal atrial lead positioning to detect retrograde atrial depolarization by digitization and slope analysis of the atrial electrogram. PACE 1984; 7:1152-1158.

21. Davies DW, Wainwright RJ, Tooley MA, et al Electrogram recognition by digital analysis: Relevance to pacemaker arrhythmia control? (abstract) J Am Coll Cardiol 1985; 5:507.
22. Davies DW, Wainwright RJ, Tooley MA, et al. Detection of pathological tachycardia by analysis of electrogram morphology. PACE 1986; 9:200-208.

23. Davies DW, Tooley MA, Cochrane T, et al. Realtime tachycardia diagnosis using morphological analysis of electrograms. (abstract) PACE:1987; 10:998.

24. DiCarlo L, Lin D, and Jenkins JM. Analysis of time and frequency domain patterns to distinguish ventricular tachycardia from sinus rhythm using endocardial electrograms. Circulation 1987; 76;280. (Abstract)

25. Throne RD, Jenkins JM, Winston SA, et al. Derivative area method: A new technique for detecting ventricular tachycardia. (abstract) Circulation 1989; 80:658.

26. Throne RD, Jenkins JM, Winston SA, et al. Discrimination of retograde from anterograde atrial activation using intracardiac electrogram waveform analysis. PACE 1989; 12:1622-1630. 
This document is a scanned copy of a printed document. No warranty is given about the accuracy of the copy. Users should refer to the original published version of the material. 